\title{
Pharmacotherapy of Bipolar Disorder with Quetiapine: A Recent Literature Review and an Update
}

\author{
Ather Muneer \\ Islamic International Medical College, Riphah International University, Rawalpindi, Pakistan
}

Bipolar disorder is a chronic, recurrent condition with the usual onset during adolescence or early adulthood. In the Diagnostic and Statistical Manual of Mental Disorders 5th edition, it is conceptualized as a spectrum disorder usually associated with such comorbidities as anxiety disorders and substance use disorders. It is a relatively prevalent condition often complicated by mixed episodes, rapid cycling, subsyndromal symptoms, and treatment refractoriness. In spite of carrying substantial morbidity and mortality, effective treatments are few and far between and conventional mood stabilizers are often unsuccessful in controlling the various manifestations of the disorder. In this scenario, second generation antipsychotics are emerging as treatments with valid efficacy in all phases of bipolar disorder. Quetiapine is a versatile atypical antipsychotic which was first approved for the treatment of schizophrenia, but latter on the basis of controlled studies earned United States Food and Drug Administration's approval for acute as well as maintenance treatment of this difficult to treat condition. In this review, recently published studies in the last 10 years were examined to update the knowledge about the efficacy and safety of quetiapine in the treatment of bipolar disorder. The medication's clinical pharmacology was first considered followed by a literature review summarizing its uses in bipolar disorder. The conclusion was that quetiapine was efficacious in manic, mixed and depressive episodes and as a maintenance agent with a good tolerability profile.

KEY WORDS: Bipolar disorder; Bipolar depression; Mania; Atypical antipsychotic agent; Mood stabilizer

\section{INTRODUCTION}

Bipolar disorders (BD) are a collection of heterogeneous conditions characterized by marked variations in mood. ${ }^{1)}$ Patients with BD lie on a spectrum; according to DSM-5 those who experience manic episodes in addition to major depressive episodes (MDE) are said to suffer from BD type I, while patients who have recurrent hypomanic episodes along with MDEs are considered as having BD type II. Two other subtypes are recognized in the Diagnostic and Statistical Manual of Mental Disorders 5th edition (DSM-5), i.e., cyclothymic disorder, a long standing condition marked by subtle mood variations and characterized by hypomanic episodes and depressive episodes which fall short of MDE and BD not elsewhere defined, a residual condition in which the clinician suspects BD but does not have enough information to make a definitive

\footnotetext{
Received: October 10, 2014 / Revised: November 19, 2014

Accepted: November 20, 2014

Address for correspondence: Ather Muneer, MD

Islamic International Medical College, Riphah International

University, 274 Peshawar Road, Rawalpindi 4622, Pakistan

Tel: +92-0515481828, Fax: +92-0515125170

E-mail: muneerather2@gmail.com
}

\section{diagnosis. ${ }^{2)}$}

Bipolar spectrum disorders have a high prevalence in the general population of about $4 \%$ and according to World Health Organization are among the ten leading causes of disability in the world. ${ }^{3)} \mathrm{BD}$ has one of the highest rates of suicide among all psychiatric conditions as many patients suffer from severe hopelessness and thoughts of dying during depressive and mixed episodes. ${ }^{4)}$ There are as yet no biomarkers for the diagnosis of this condition and the clinician has to rely on history and mental state examination to make an empirical diagnosis. BD is a chronic, lifelong condition with the usual age of onset in adolescence or early adulthood. ${ }^{5)}$ There is no definitive treatment and therapeutic agents only decrease the burden of symptoms to varying degrees. ${ }^{6}$ )

Risperidone was the first atypical antipsychotic which was approved by the United States Food and Drug Administration (FDA) in 1994 for the treatment of acute schizophrenia. It was followed by other atypical or second generation antipsychotics (SGA) namely olanzapine, quetiapine, ziprasidone, aripiprazole and more recently iloperidone, asenapine, and lurasidone. SGAs differ from

() This is an Open-Access article distributed under the terms of the Creative Commons Attribution Non-Commercial License (http://creativecommons.org/licenses/by-nc/3.0) which permits unrestricted non-commercial use, distribution, and reproduction in any medium, provided the original work is properly cited. 
typical antipsychotics in a number of ways; firstly they bind to the dopamine $\mathrm{D}_{2}$ receptor with less affinity, secondly they exert their action predominantly in the mesolimbic and mesocortical dopamine pathways as opposed to the nigrostraital pathway, and lastly they antagonize the $5 \mathrm{HT}_{2 \mathrm{~A}}$ serotonin receptor - a pharmacodynamic property that the typical antipsychotics do not possess. ${ }^{7)}$ The net result is that SGAs cause less extrapyramidal side effects and have valid efficacy in a host of other neuropsychiatric conditions including mood disorders, anxiety spectrum disorders, and stressor-related disorders. ${ }^{8)}$

Quetiapine first received FDA approval in 1997 for the treatment of acute episodes of schizophrenia in adults. The pharmacological properties of this medication soon led to uses in other neuropsychiatric conditions in particular affective disorders, anxiety disorders, autism spectrum disorders, dementia and delirium. ${ }^{9)}$ Efficacy in double blind, randomized, controlled trials was the basis for the licensing of quetiapine in bipolar mania, bipolar depression, bipolar maintenance and major depressive disorder either as monotherapy or adjunctive to other medications. ${ }^{10)}$ Now quetiapine has become one of the most commonly prescribed drugs for bipolar patients and is used in all phases of this disorder. ${ }^{11)}$ The purpose of this paper is to delineate the pharmacological properties of quetiapine and provide an update in the knowledge regarding its uses in $\mathrm{BD}$.

\section{MAIN SUBJECTS}

\section{Pharmacology of Quetiapine}

\section{Pharmacokinetics}

Since 2006 quetiapine has been available as immediate-release (IR) and extended-release (XR) formulations. When administered in a dose within the therapeutic range the drugs show linear kinetics with an elimination half-life of approximately 7 hours. Both IR and XR formulations provide equal bioavailability; however, the time to attainment of peak plasma concentration is 5 hours for XR and 2 hours for IR. Moreover, higher plasma levels are sustained for a longer period of time with quetiapine XR, hence once daily dosing of the XR formulation is required to maintain therapeutic drug concentrations as opposed to IR which needs to be administered at least twice daily. ${ }^{12)}$

Quetiapine is extensively metabolized by the liver into various metabolites with only $1 \%$ excreted unaltered in the urine. $N$-desalkyl quetiapine or norquetiapine is quetiapine's key metabolite and is produced by the action of isoenzymes CYP 3A4 in the cytochrome P450 system. ${ }^{13)}$ Minor metabolism occurs via CYP2D6 into 7-hydroxy quetiapine which is thought not to possess any active properties. The efficacy of quetiapine in treating psychotic disorders as well as mood and anxiety disorders leads to the view that it is a multifunctional psychoactive drug. Its broad spectrum of efficacy is likely due to its ability to modify systems of dopaminergic, serotonergic and noradrenergic neurotransmission and its effects appear to be mediated by the actions of both quetiapine and norquetiapine. ${ }^{14)}$

\section{Pharmacodynamic profile}

The blockade of dopamine $\mathrm{D}_{2}$ receptor in the mesolimbic pathway is considered to be the main mechanism behind antipsychotic efficacy. Both quetiapine and norquetiapine bind with moderate affinity to $\mathrm{D}_{1}$ and $\mathrm{D}_{2}$ receptors, additionally the former rapidly dissociates from $\mathrm{D}_{2}$ receptors explaining the need for administration of high doses of quetiapine to bring about its antipsychotic effect. ${ }^{15)}$ It also exhibits low capacity for up-regulation of these receptors, which explains the low incidence of tardive dyskinesia associated with prolonged quetiapine therapy. In the nigrostriatal and tuberoinfundibular dopamine pathways serotonin seems to act as an inhibitory modulator by its action on $5 \mathrm{HT}_{2 \mathrm{~A}}$ receptor. Both quetiapine and norquetiapine strongly antagonize this receptor, thereby easing dopamine release in the said pathways and resulting in low incidence of extrapyramidal side effects and hyperprolactinemia. ${ }^{16)}$

Many symptoms of depression like anhedonia, psychomotor retardation, social withdrawal and loss of motivation result from decreased dopamine neurotransmission in the prefrontal cortex (PFC). It is believed that norquetiapine with its $5 \mathrm{HT}_{2 \mathrm{~A}}$ and $5 \mathrm{HT}_{2 \mathrm{C}}$ antagonism facilitates dopamine release in PFC and is instrumental in relieving the depressive symptoms in patients with mood disorders. ${ }^{17)}$ Dopamine reuptake in the PFC is mediated by norepinephrine transporter; norquetiapine and not quetiapine is a strong inhibitor of this transporter, thereby adding another mechanism to the parent drug's antidepressant efficacy. ${ }^{18)}$

Quetiapine, and to a greater extent its metabolite norquetiapine facilitate serotonergic transmission by behaving as partial agonists at $5 \mathrm{HT}_{1 \mathrm{~A}}$ receptors which are associated with antidepressant and anxiolytic effects in humans. Norquetiapine has a high affinity for $5 \mathrm{HT}_{1 \mathrm{~A}}$ receptors, similar to that of conventional agonists' buspirone and gepirone. Through this mechanism it increases serotonergic neurotransmission by the raphe neurons in the 
brain stem as well as modulates 5HT functioning in the limbic and cortical regions. ${ }^{19)}$ By activating the $5 \mathrm{HT}_{1 \mathrm{~A}}$ receptors in the hippocampus, norquetiapine can spur neuron regeneration by increasing the release of trophic factors like the brain-derived neurotrophic factor. ${ }^{20)}$ Norquetiapine also has a higher affinity for $5 \mathrm{HT}_{7}$ receptor than quetiapine. This receptor's involvement in depression and sleep-related, circadian rhythm disorders has been experimentally documented, and it is possible that norquetiapine's $5 \mathrm{HT}_{7}$ receptor antagonism contributes to quetiapine's antidepressant action. ${ }^{21)}$

\section{Adverse effects}

The receptor binding profile of quetiapine and its active metabolite norquetiapine explains the adverse effects of these substances. Both of them bind to histamine H1 receptors causing sedation, hypnotic effects, increased appetite and weight gain. Blockage of alpha 1 receptors by both these compounds explains the orthostatic hypotension experienced by some patients. Norquetiapine acts as a strong antagonist at muscarinic $\mathrm{M}_{1}, \mathrm{M}_{3}$, and $\mathrm{M}_{5}$ receptors so that anticholinergic effects like dry mouth, urinary retention, pupillary dilatation, raised intraocular pressure, and hypothermia result from the action of this active metabolite. Some researchers are of the view that hyperglycemia and diabetes caused by quetiapine therapy are primarily the result of $\mathrm{M}_{3}$ receptor antagonism. ${ }^{22)}$ So it would appear that while norquetiapine is responsible for therapeutic effects it may also be to blame for quetiapine's adverse effects.

\section{Dosage}

During therapy, quetiapine's dose can be rapidly titrated within 2 to 3 days to the maximum recommended without fear of causing serious adverse effects. While treating acute schizophrenia, the recommended initial dose on day 1 is $300 \mathrm{mg}$ which can be increase by $200 \mathrm{mg}$ to $300 \mathrm{mg}$ per day to a maximum dose of $800 \mathrm{mg}$ per day. For schizophrenia maintenance the recommended daily dose is $400-800 \mathrm{mg} /$ day. For bipolar mania the dosage instruction states that $300 \mathrm{mg}$ be administered on day 1,600 $\mathrm{mg}$ on day 2 and up to a maximum of $800 \mathrm{mg}$ on day 3 , while the dosage range remains $400-800 \mathrm{mg} /$ day. $^{23}$ )

In the treatment of depressive episodes associated with BD start with a dose of $50 \mathrm{mg}$ on day 1 , increased to 100 $\mathrm{mg}$ on day 2,200 $\mathrm{mg}$ on day 3 and $300 \mathrm{mg}$ on day 4 maximum recommended dose being $300 \mathrm{mg} /$ day. For bipolar I maintenance, as an adjunct to lithium or divalproex the dosage range of quetiapine as per instructions is $400-800$ $\mathrm{mg} /$ day.

When using quetiapine in major depressive disorder as an adjunct to an antidepressant, the starting dose is $50 \mathrm{mg}$ on day 1 and day 2. The dose can be increased to $150 \mathrm{mg}$ on day 3 and day 4, while the recommended daily dosage in the adjunctive therapy of major depressive disorder is $150-300 \mathrm{mg} /$ day. $^{24)}$

\section{Advances in the Pharmacotherapy of Bipolar Disorder}

Atypical antipsychotics were introduced for the treatment of psychotic disorders such as schizophrenia and these drugs were of equal efficacy to conventional antipsychotics in this regard. The former had a different profile, in that these were more effective in dealing with the negative and cognitive symptoms of schizophrenia and were associated with less medication induced extrapyramidal side-effects including tardive dyskinesia. ${ }^{25)}$ Soon after their launch, clinical evidence pointed towards the efficacy of SGA in the treatment of BD, either as adjunct to classical mood stabilizers or as monotherapy. ${ }^{26)}$

The addition of typical antipsychotics to mood stabilizers resulted in a more rapid onset of action and in better control of agitation and psychotic behavior during manic episodes of BD. Conventional antipsychotics were effective antimanic agents, however these had no efficacy against the depressive symptoms; rather their long term use in BD was associated with the induction of secondary depressive symptoms and as such this practice was usually discouraged. Several studies suggested that SGAs were effective not only in the treatment of acute mania but also in the management of other phases of BD. ${ }^{27)}$ For example, olanzapine monotherapy was as effective as classical mood stabilizers (lithium, divalproex) in the maintenance treatment of BD. Additionally, olanzapine in combination with fluoxetine, as well as olanzapine monotherapy were found to be more effective than placebo in the treatment of bipolar depression. Latter studies on other atypical antipsychotics provided evidence of efficacy for quetiapine, aripiprazole and lurasidone in the depressive as well as maintenance phases of BD. ${ }^{28)}$

As more was known about the pharmacodynamic properties of SGAs, it became apparent that these agents had efficacy in the treatment of all phases of BD by virtue of their receptor binding properties. In particular, the affinity for different $5 \mathrm{HT}$ receptors appeared to play a crucial role in the mood stabilizing characteristic of these medications. ${ }^{29)}$ As a result of this receptor binding there were secondary changes in the intracellular signal transduction pathways and activity of nerve growth factors, effects that 
were similar to classical mood stabilizers like lithium. ${ }^{30)}$

\section{Quetiapine as a Mood Stabilizer}

For the successful pharmacological management of BD the following objectives must be achieved:

a) Control of manic and mixed episodes,

b) Treatment of depressive episodes and sub threshold depressive symptoms,

c) Avoidance of switching into mania during anti-depressant treatment and,

d) Stabilization of mood in between episodes to avoid relapses and recurrences.

Conventional mood stabilizers such as lithium and anticonvulsants represented the treatment of first choice to achieve the above-mentioned goals but carried a number of disadvantages. These, so called classical mood stabilizers had limited clinical efficacy in severe manic and mixed episodes, bipolar depression and as prophylactic agents. Additionally these had a narrow therapeutic index and required frequent plasma determinations to avoid toxicity and adverse effects. ${ }^{31)}$

In contrast, quetiapine represented advancement in each one of these areas. It was successful in treating severe manic and mixed episodes either as monotherapy or adjunctive to conventional mood stabilizers. The same was true in the therapy for bipolar depression and during maintenance treatment. ${ }^{32)}$ The drug had a low side effect profile, was generally well tolerated and represented no need for therapeutic plasma monitoring.

A retrospective study conducted to evaluate the effect of adjunctive quetiapine, included 16 patients with bipolar I disorder (nine were in a mixed episode, others were manic) who were on monotherapy with lithium or valproate for a minimum of 3 months prior to quetiapine initiation. BD symptoms were evaluated using the Clinical Global Impression Scale for severity of bipolar illness (CGI-BP), at baseline and at endpoint after quetiapine dose had been stable for at least one month. Considering CGI-BP severity scores, the addition of quetiapine resulted in a significant improvement in mania, depression, and overall bipolar illness. Quetiapine efficacy was particularly evident on insomnia, psychomotor agitation, depressed mood, elevated mood, racing thoughts, irritability, impulsivity, pressured speech, hypersexuality and psychosis. ${ }^{33)}$

\section{Quetiapine Treatment of Mania and Mixed States}

A summary of the studies depicted in this section is provided in Table 1.

In a paper published in 2013, the efficacy and safety of

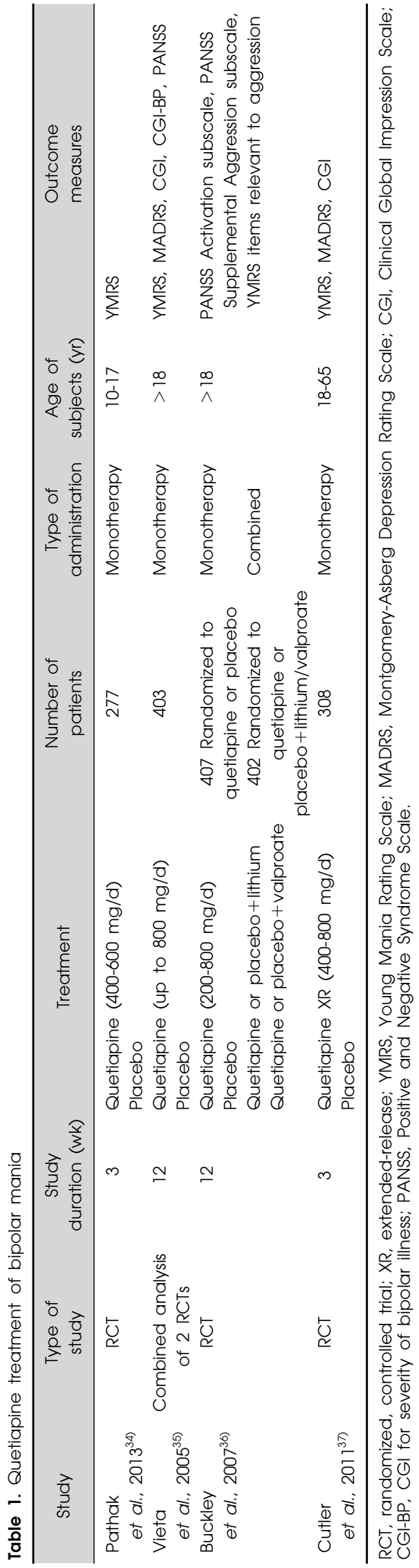


quetiapine monotherapy was studied in children and adolescents with bipolar I mania in a 3 week, double blind, placebo-controlled, multicenter trial. Patients aged 10 to 17 years, with the Diagnostic and Statistical Manual of Mental Disorders 4th edition, text revision (DSM-IV TR) diagnosis of a manic episode (bipolar I disorder) and Young Mania Rating Scale (YMRS) total score equal to or greater than 20 were randomized to 3 weeks of quetiapine (400 or $600 \mathrm{mg} /$ day) or placebo. The primary efficacy measure was change in YMRS total score. The intent-to-treat population included 277 patients from 34 centers in the United States. Mean change in YMRS score from baseline to end point at 3 weeks was -14.25 , -15.60 , and -9.04 for quetiapine $400 \mathrm{mg} /$ day, quetiapine $600 \mathrm{mg} /$ day and placebo respectively. Mean changes in body weight at day 21 were $1.7 \mathrm{~kg}$ for both doses of quetiapine and $0.4 \mathrm{~kg}$ for placebo. Numerically larger mean increases in total cholesterol, low-density lipoprotein cholesterol, and triglycerides were observed with quetiapine than placebo. Adverse events associated with quetiapine were mostly mild to moderate in intensity. The authors concluded that in this 3-week study, quetiapine was significantly more effective than placebo in improving manic symptoms in youth with mania associated with BD. Treatment was generally well tolerated and adverse events were broadly consistent with the known profile of quetiapine in adults with BD. ${ }^{34)}$

In a landmark study, combined analysis was done of two international, double-blind, randomized, placebo-controlled trials evaluating the efficacy and safety of quetiapine monotherapy in patients with acute bipolar mania. The patients were randomized to 12 weeks of treatment with quetiapine (up to $800 \mathrm{mg} /$ day) or placebo. The primary efficacy end point was change in YMRS score from baseline to day 21. Secondary end points included change from baseline in YMRS to day 84, YMRS response and remission rates and change from baseline to days 21 and 84 in the Montgomery-Asberg Depression Rating Scale (MADRS), CGI, CGI-BP and Positive and Negative Syndrome Scale (PANSS). The combined analysis included a total of 403 patients from two quetiapine monotherapy studies in patients with bipolar I disorder. A significant improvement in YMRS score was observed from day 4 ( $p=0.021)$ in the quetiapine group compared with placebo. The treatment advantage of quetiapine over placebo continued to increase to day $21(p<0.001)$ and day $84(p<0.001)$. Significantly more quetiapine-treated than placebo-treated patients achieved a response $(p<0.001)$. The average quetiapine dose in responders was approx- imately $600 \mathrm{mg}$ daily. Of adverse events occurring in $\geq 5 \%$ of patients, quetiapine-treated patients had significantly greater incidence versus placebo of somnolence ( $16.3 \%$ vs. $4 \%)$, dry mouth $(15.8 \%$ vs. $3 \%)$, weight gain $(9.1 \%$ vs. $1.5 \%)$ and dizziness $(6.7 \%$ vs. $2.5 \%)$. The data from this combined analysis supported the results from the individual studies and indicated that quetiapine monotherapy was effective across a broad range of mood symptoms, fast acting and well tolerated in the treatment of mania. ${ }^{35)}$

In another paper published few years ago, quetiapine's efficacy was studied in the treatment of aggression and agitation in bipolar mania which were potentially disruptive and dangerous features of this disorder. Four hundred and seven patients with bipolar I mania were randomized to quetiapine monotherapy (200-800 mg/day) or placebo for 12 weeks, and 402 patients were randomized to quetiapine (200-800 mg/day) or placebo in combination with lithium (Li) or divalproex (DVP) for 3 or 6 weeks. Measurements of agitation included the PANSS Activation subscale, PANSS Supplemental Aggression Risk subscale scores, and YMRS items relevant to agitation. The results showed that initial reductions in both the PANSS Activation and PANSS Supplemental Aggression Risk subscale scores were noted by day 4 with quetiapine and placebo. The reduction in PANSS Activation subscale scores was significantly greater with quetiapine monotherapy than placebo first at day 21 and also at day 84 . The improvement in PANSS Supplemental Aggression Risk subscale score was significantly greater with quetiapine (QTP) monotherapy than placebo (PBO) by day 14 and at all time points thereafter including day 21 and day 84 . In combination therapy, the mean improvement in PANSS Activation subscale score at day 21 was numerically but not significantly different with QTP $+\mathrm{Li} / \mathrm{DVP}$ than $\mathrm{PBO}+$ Li/DVP. The mean PANSS Supplemental Aggression Risk subscale scores were significantly improved at day 21 with QTP $+\mathrm{Li} / \mathrm{DVP}$ versus $\mathrm{PBO}+\mathrm{Li} / \mathrm{DVP}$. The study clearly showed that quetiapine was an effective and appropriate treatment choice in managing agitation and aggression associated with bipolar mania. ${ }^{36}$

A randomized, double blind, 3-week phase III trial evaluated the efficacy and tolerability of once-daily extended-release quetiapine fumarate monotherapy in improving manic symptoms in patients with BD type I. Patients aged 18 to 65 years with bipolar I disorder (most recent episode manic or mixed, with or without rapid cycling) were randomized to receive placebo or quetiapine XR monotherapy once daily (300 mg on day 1; $600 \mathrm{mg}$ on day 2; flexible dosing $400 \mathrm{mg}$ to $800 \mathrm{mg}$ from day 3 
through day 22 [study end point week 3]). The primary outcome measure was the change from baseline to study end in the YMRS total score. Secondary outcome measures included the MADRS total score, YMRS response (greater than 50\% reduction in YMRS) and remission (YMRS score less than 12 at final visit) rates, and change from baseline to week 3 in Clinical Global ImpressionBipolar-Severity of illness (CGI-BP-S) and CGI-BPChange (CGI-BP-C) scores. Safety profile and tolerability evaluations included monitoring of adverse events, clinical laboratory values, vital signs, extrapyramidal symptoms (including akathisia), and electrocardiogram results.

The results showed that compared with placebo ( $\mathrm{n}=159)$, quetiapine XR monotherapy $(\mathrm{n}=149$; mean daily dose $604 \mathrm{mg}$ ) significantly improved manic symptoms starting at day 4 with sustained improvement to study end at week 3. MADRS scores showed greater improvement from baseline to study end at day 22 with quetiapine XR than with placebo. Response and remission rates were significantly greater with quetiapine XR than with placebo at study end. Quetiapine XR also resulted in significant improvement over placebo in CGI-BP-S and CGI-BP-C scores. Adverse events were mild to moderate in intensity; the most common ones associated with quetiapine XR were sedation, dry mouth and somnolence. The authors concluded that this 3 week trial suggested that quetiapine XR (400-800 mg) once daily monotherapy was efficacious from day 4 and generally well tolerated in patients with manic or mixed episodes of bipolar I disorder. ${ }^{37)}$

\section{Quetiapine Treatment of Bipolar Depression}

Studies on the longitudinal course of BD showed that patients spent much more time suffering from depressive symptoms as compared to manic symptoms. ${ }^{38)}$ The former could be in the form of acute MDE which might require hospitalization or chronic sub threshold symptoms with complex comorbidities like anxiety spectrum disorders, substances use disorders, eating disorders and stressorrelated disorders. ${ }^{39)}$ A very large number of patients from $25 \%$ to $55 \%$ made medically serious suicide attempt, and $10 \%$ to $20 \%$ were successful in committing suicide, representing a huge burden on the sufferers of $\mathrm{BD} .{ }^{40)}$ With these statistics in mind it was imperative to find effective treatments for the depressive phase of BD. A number of studies showed that quetiapine monotherapy was an efficacious treatment for acute bipolar depression at least in adult patients of both sexes.

Table 2 gives an outline of the studies of quetiapine in bipolar depression described in this part of the paper. 
The EMBOLDEN I study compared the efficacy and tolerability of quetiapine and lithium monotherapy with that of placebo for a MDE in BD. Eight hundred and two patients with DSM-IV defined BD (499 bipolar I, 303 bipolar II) were randomly allocated to quetiapine 300 $\mathrm{mg} /$ day $(\mathrm{n}=265)$, quetiapine $600 \mathrm{mg} /$ day $(\mathrm{n}=268)$, lithium $600-1,800 \mathrm{mg} /$ day $(\mathrm{n}=136)$, or placebo $(\mathrm{n}=133)$ for 8 weeks. Primary endpoint was the change in MADRS total score. The study was conducted from August 2005 to May 2007.

The results showed that the mean MADRS total score change from the baseline at 8 weeks was -15.4 for quetiapine $300 \mathrm{mg} /$ day, -16.1 for quetiapine $600 \mathrm{mg} /$ day, -13.6 for lithium, and -11.8 for placebo. Quetiapine $600 \mathrm{mg} /$ day was significantly more effective than lithium in improving MADRS total score at week 8. Quetiapine treated (both doses), but not lithium treated, patients showed significant improvements $(p<0.05)$ in MADRS response and remission rates, Hamilton Rating Scale for Depression (HRSD), Clinical Global Impressions-Severity of illness and -Change, Hamilton Rating Scale for anxiety (HAM-A) scores at week 8 versus placebo. Both quetiapine doses were more effective than lithium on the HRSD and HAM-A at week 8 . The study concluded that quetiapine (300 or $600 \mathrm{mg} /$ day) was more effective than placebo for the treatment of acute episodes of depression in BD. Lithium did not differ significantly from placebo on the main measures of efficacy. ${ }^{41)}$

The EMBOLDEN II study aimed to evaluate the efficacy and tolerability of quetiapine and paroxetine monotherapy for major depression in BD. Seven hundred and forty patients (478 bipolar I and 262 bipolar II) with MDE were randomly assigned to quetiapine $300 \mathrm{mg} /$ day $(\mathrm{n}=245)$, quetiapine $600 \mathrm{mg} /$ day $(\mathrm{n}=247)$, paroxetine 20 $\mathrm{mg} /$ day $(\mathrm{n}=122)$, and placebo $(\mathrm{n}=126)$ for 8 weeks. The primary endpoint was the change from baseline in MADRS total score. The study was conducted from May 2005 to May 2007.

The results showed that the mean MADRS score change from baseline at 8 weeks was -16.19 for quetiapine $300 \mathrm{mg} /$ day, -16.31 for quetiapine $600 \mathrm{mg},-13.76$ for paroxetine, and -12.60 for placebo. Quetiapine treated (both doses), but not paroxetine-treated, patients showed significantly greater improvement $(p \leq 0.05)$ in most secondary outcome measures at week 8 versus the placebo group. Paroxetine significantly improved HAM-A scores versus placebo $(p<0.05)$ but not MADRS or HRSD scores. Both quetiapine doses were associated with greater improvement than paroxetine for MADRS and
HRSD scores. The most common adverse effects were dry mouth, somnolence, sedation, and dizziness for quetiapine (both doses) and dry mouth, sedation, headache, insomnia, and nausea with paroxetine. The incidence of treatment-emergent mania/hypomania was lower with quetiapine compared with paroxetine and placebo. The conclusions were that quetiapine (300 or $600 \mathrm{mg}$ /day), but not paroxetine, was more effective than placebo for treating acute depressive episodes in bipolar I and II disorder. Quetiapine treatment was generally well tolerated. ${ }^{42)}$

Another double blind, placebo-controlled study evaluated the effectiveness of quetiapine extended release once daily in bipolar depression. The subjects were acutely depressed adults with bipolar I or II disorder, with or without rapid cycling. Patients were randomized to 8 weeks of quetiapine XR $300 \mathrm{mg}$ daily monotherapy or placebo. The primary outcome measure was change from baseline to Week 8 in MADRS total score.

The results showed that quetiapine XR $300 \mathrm{mg}$ once daily ( $\mathrm{n}=133$ ) showed significantly greater improvement in depressive symptoms compared with placebo $(n=137)$ from week 1 through to week 8. Mean change in MADRS total score at week 8 was -17.4 in the quetiapine $\mathrm{XR}$ group and -11.9 in the placebo group $(p<0.001)$. Response ( $\geq 50 \%$ reduction in MADRS total score) and remission (MADRS total score $\leq 12$ ) rates at week 8 were significantly higher with quetiapine XR compared with placebo. Quetiapine XR improved core symptoms of depression. The study concluded that quetiapine XR (300 $\mathrm{mg}$ ) once daily monotherapy was significantly more effective than placebo for treating episodes of acute depression in $\mathrm{BD}$, throughout the 8-week study, with significance observed as early as day 7 . Adverse events were consistent with the known effects of quetiapine. ${ }^{43}$

A recently published meta-analysis systematically reviewed the efficacy and tolerability of quetiapine either as monotherapy or adjunctively to mood stabilizers in the treatment of acute bipolar depression. The authors included all randomized, controlled trials (RCTs) comparing quetiapine with other treatments including placebo, in patients with MDEs associated with bipolar I and bipolar II disorders. Published and unpublished RCTs were identified using the Cochrane Central Register of Controlled Trials, MEDLINE, Web of Knowledge, CINHAL, PsycINFO, the EU Clinical Trials Register Database, and ClinicalTrials.gov. The primary outcome was the change in scores of depression rating scales. Fifteen hundred and twelve records were identified through database searching out of which 1,017 records were screened after removal of 
duplicates. Nine hundred and ninety two records were excluded because of methodological reasons and of the remaining 25 studies, full text articles were assessed for eligibility. A further 14 full text records were excluded because these did not entirely fulfill the eligibility criteria for RCTs while meta-analysis was performed on the remaining eleven studies.

The number of patients included in these 11 RCTs was 3,488 and two of the trials were conducted in children and adolescents (ages 10 to 18). The change in depression scores was significantly greater in the quetiapine group compared with the placebo group (mean difference $=-$ $4.66,95 \%$ confidence interval $[\mathrm{CI}]-5.59$ to -3.73 ). The significant difference was observed from week 1 . Quetiapine treatment was associated with significant improvement of clinical global impression, quality of life, sleep quality, anxiety and functioning. The conclusion was that quetiapine monotherapy was effective for acute bipolar depression and the prevention of mania/hypomania switching. The most common adverse events were extrapyramidal reactions, sedation, somnolence, dizziness, fatigue, constipation, dry mouth, increased appetite, and weight gain. The evidence for the use of quetiapine combined with mood stabilizers in children and adolescents with acute bipolar depression was too small to support the clinical practice. ${ }^{44)}$

\section{Quetiapine in Bipolar Maintenance}

For a summary of the studies illustrated in this section see Table 3.

$\mathrm{BD}$ is a highly recurrent condition with relapses that could be depressive, manic or mixed in nature. There are few approved treatments to prevent recurrences in $\mathrm{BD}$; one such option is quetiapine adjunctive to lithium or divalproex sodium for the maintenance treatment of $\mathrm{BD}$. In an analysis of two large randomized, placebo-controlled trials the safety and efficacy of quetiapine combined with lithium or divalproex for preventing mood events in patients with bipolar I disorder was determined. In this pooled analysis, patients received open label quetiapine (400-800 mg/day) plus lithium or divalproex to achieve at least 12 weeks of clinical stability before being randomized to double-blind combination treatment with quetiapine (400-800 mg/day) or placebo plus lithium or divalproex for up to 104 weeks. The primary endpoint was time to first mood event post randomization following open stabilization.

The results of the analysis showed that of the 3,414 patients in the stabilization phase, 1,326 were randomized.

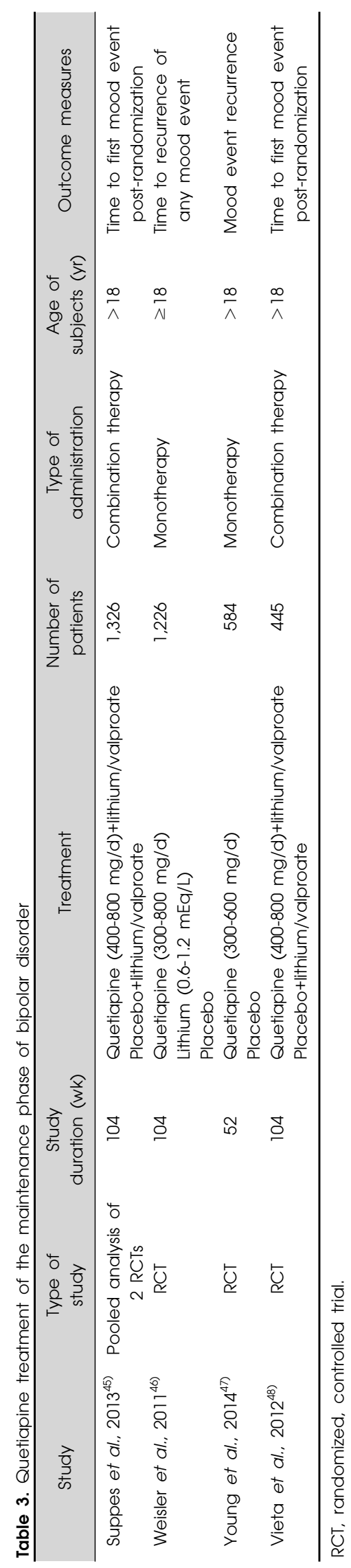


There were no differences in the risk of recurrence of mood events, mania or depression between quetiapine plus lithium or quetiapine plus divalproex. Among patients co-treated with placebo and lithium, the risk of recurrence of a mania event was significantly higher than among patients co-treated with placebo and divalproex. In patients with an index episode of mania, placebo plus lithium was associated with a significantly higher risk of recurrence of a mania event than placebo plus divalproex. Safety data were generally consistent with recognized safety profiles. The study concluded that in patients with bipolar I disorder previously stabilized on quetiapine and lithium or divalproex, maintenance therapy with quetiapine significantly increased time to recurrence of a mood event (mania or depression) versus placebo, regardless of whether it was combined with lithium or divalproex. ${ }^{45}$ )

A controlled study investigated the efficacy and safety of quetiapine monotherapy as maintenance treatment in bipolar I disorder compared with switching to placebo or lithium. Patients aged $\geq 18$ years with DSM-IV-diagnosed bipolar I disorder and a current or recent manic, depressive, or mixed episode received open-label quetiapine (300-800 mg/day) for 4 to 24 weeks. Patients achieving stabilization were randomized to continue quetiapine or to switch to placebo or lithium $(0.6-1.2 \mathrm{mEq} / \mathrm{L})$ for up to 104 weeks in a double-blind trial. Outcome measures included times to recurrence of any mood event (primary outcome measure), manic event, or depressive event. Safety assessments included adverse events and laboratory values. The study was terminated early after planned interim analysis provided positive results. The study was conducted between March 2005 and July 2007.

Of the 2,438 patients starting open-label quetiapine, $1,226(50.3 \%)$ were randomized to double-blind treatment, including $1,172(95.6 \%)$ in the intent-to-treat population. Time to recurrence was significantly longer for quetiapine versus placebo (hazard ratio $[\mathrm{HR}]=0.29 ; 95 \%$ CI $0.23-0.38 ; p<0.0001)$ and for lithium versus placebo (HR $=0.46 ; 95 \%$ CI $0.36-0.59 ; p<0.0001$ ). Quetiapine and lithium significantly increased time to recurrence of both manic events and depressive events compared with placebo. Overall rates of adverse events were generally similar between treatment groups, and safety findings for quetiapine were consistent with its known profile. The conclusion was that in patients stabilized during acute quetiapine treatment, continuation of quetiapine significantly increased time to recurrence of any mood event compared with switching to placebo. Switching to lithium was also more effective than placebo for the prevention of manic and depressive events. ${ }^{46}$

A recently published study examined the longer-term efficacy of quetiapine monotherapy in bipolar depression in a preplanned pooling of data from the EMBOLDEN I AND II studies. Patients ( $\mathrm{n}=584)$ with bipolar I or II disorder (most recent episode: depressed) who achieved remission after 8 weeks of treatment with quetiapine (300 or $600 \mathrm{mg} /$ day) were randomized to the same quetiapine dose or placebo for 26-52 weeks or until mood event recurrence. The risk for recurrence of a mood event was significantly lower with quetiapine than placebo. Quetiapine was associated with a lower risk for recurrence of depressive events but recurrence of manic/hypomanic events was not significantly reduced. There was a lower risk of recurrence of mood events in bipolar I and bipolar II patients. Discontinuation rates were 4.3, 4.0 and 1.7\% for quetiapine $300 \mathrm{mg} / \mathrm{day}, 600 \mathrm{mg} / \mathrm{day}$ and placebo, respectively. Safety data, including changes in lipid and glucose parameters, were consistent with the recognized profile of quetiapine. The conclusions were that the efficacy of quetiapine monotherapy in bipolar depression was maintained during continued treatment for 26-52 weeks. Quetiapine treatment was generally well tolerated. ${ }^{47)}$

Another study evaluated quetiapine in patients with bipolar I disorder with mixed symptoms. Data from 2 studies (D1447C00126, D1447C00127) were pooled and mixed events analyzed separately. Patients received quetiapine (400-800 mg/day) plus lithium/divalproex to achieve at least 12 weeks of clinical stability, followed by double-blind quetiapine $(400-800 \mathrm{mg} /$ day $)$ or placebo plus lithium/divalproex, for up to 104 weeks. Primary endpoint was time to first mood event post-randomization. The total number of patients was 1,326, of whom 445 had a mixed episode at study entry, 219 received quetiapine plus lithium/divalproex, and 226 received placebo plus lithium/divalproex. Mood events were reported by fewer quetiapine-plus-lithium/divalproex than placebo-plus-lithium/divalproex-treated patients $(21 \%$ vs. $54 \%$ ), and included mixed ( $6.4 \%$ vs. $22.1 \%$ ), pure manic $(5.0 \%$ vs. $13.3 \%)$, and pure depressed events $(9.6 \%$ vs. $18.6 \%$ ). HR for time to recurrence were longer for quetiapine plus lithium/divalproex than placebo plus lithium/divalproex for all mood events. This post-hoc analysis showed that in stable patients with bipolar I disorder, quetiapine plus lithium/divalproex significantly increased time to recurrence of mood events versus placebo in patients with mixed symptoms at study entry and time to occurrence of mixed-mood events in patients with any mood episode at study entry. ${ }^{48)}$ 


\section{CONCLUSION}

Quetiapine is a SGA which was first introduced for the treatment of acute schizophrenia. Latter, on the basis of double-blind, placebo-controlled trials it was found to be an effective treatment for BD. The drug had valid efficacy in manic, mixed and depressive exacerbations either as monotherapy or as an adjunct to other mood stabilizers. The same was true in the treatment of the maintenance phase of BD. As such quetiapine was a unique psychotherapeutic agent and was FDA approved for the treatment of all phases of $\mathrm{BD}$, becoming in recent years one of the most widely prescribed drug for this disorder. As it carried the burden of metabolic side-effects, measures needed to be taken to guard against obesity, dyslipidemia and hyperglycemia; otherwise the drug was generally safe and well tolerated.

\section{REFERENCES}

1. Kurumaji A, Narushima K, Ooshima K, Yukizane T, Takeda M, Nishikawa T. Clinical course of the bipolar II disorder in a Japanese sample. J Affect Disord 2014;168:363-366.

2. Ghaemi SN, Dalley S. The bipolar spectrum: conceptions and misconceptions. Aust N Z J Psychiatry 2014;48:314-324.

3. Whiteford HA, Degenhardt L, Rehm J, Baxter AJ, Ferrari AJ, Erskine HE, et al. Global burden of disease attributable to mental and substance use disorders: findings from the Global Burden of Disease Study 2010. Lancet 2013;382: 1575-1586.

4. Clements C, Morriss R, Jones S, Peters S, Roberts C, Kapur N. Suicide in bipolar disorder in a national English sample, 1996-2009: frequency, trends and characteristics. Psychol Med 2013;43:2593-2602.

5. Fagiolini A, Forgione R, Maccari M, Cuomo A, Morana B, Dell'Osso MC, et al. Prevalence, chronicity, burden and borders of bipolar disorder. J Affect Disord 2013;148: 161-169.

6. Vieta E, Langosch JM, Figueira ML, Souery D, BlascoColmenares E, Medina E, et al. Clinical management and burden of bipolar disorder: results from a multinational longitudinal study (WAVE-bd). Int J Neuropsychopharmacol 2013;16:1719-1732.

7. Seeman P. Atypical antipsychotics: mechanism of action. Can J Psychiatry 2002;47:27-38.

8. Maher AR, Theodore G. Summary of the comparative effectiveness review on off-label use of atypical antipsychotics. J Manag Care Pharm 2012;18(5 Suppl B):S1-S20.

9. Hawkins SB, Bucklin M, Muzyk AJ. Quetiapine for the treatment of delirium. J Hosp Med 2013;8:215-220.

10. Sanford M. Quetiapine extended release: adjunctive treatment in major depressive disorder. CNS Drugs 2011;25:803-813.

11. Plosker GL. Quetiapine: a pharmacoeconomic review of its use in bipolar disorder. Pharmacoeconomics 2012;30:611-631.

12. Bui K, Earley W, Nyberg S. Pharmacokinetic profile of the extended-release formulation of quetiapine fumarate (quetiapine XR): clinical implications. Curr Med Res Opin 2013; 29:813-825.
13. López-Muñoz F, Alamo C. Active metabolites as antidepressant drugs: the role of norquetiapine in the mechanism of action of quetiapine in the treatment of mood disorders. Front Psychiatry 2013;4:102.

14. Altamura AC, Moliterno D, Paletta S, Buoli M, Dell'osso $\mathrm{B}$, Mauri $\mathrm{MC}$, et al. Effect of quetiapine and norquetiapine on anxiety and depression in major psychoses using a pharmacokinetic approach: a prospective observational study. Clin Drug Investig 2012;32:213-219.

15. Kapur S, Zipursky R, Jones C, Shammi CS, Remington G, Seeman P. A positron emission tomography study of quetiapine in schizophrenia: a preliminary finding of an antipsychotic effect with only transiently high dopamine D2 receptor occupancy. Arch Gen Psychiatry 2000;57:553-559.

16. Mundo E, Cattaneo E, Zanoni S, Altamura AC. The use of atypical antipsychotics beyond psychoses: efficacy of quetiapine in bipolar disorder. Neuropsychiatr Dis Treat 2006;2:139-148.

17. Rasmussen H, Ebdrup BH, Aggernaes B, Lublin H, Oranje $\mathrm{B}$, Pinborg LH, et al. Norquetiapine and depressive symptoms in initially antipsychotic-naive first-episode schizophrenia. J Clin Psychopharmacol 2013;33:266-269.

18. Björkholm C, Jardemark K, Marcus MM, Malmerfelt A, Nyberg S, Schilström B, et al. Role of concomitant inhibition of the norepinephrine transporter for the antipsychotic effect of quetiapine. Eur Neuropsychopharmacol 2013;23: 709-720.

19. Silverstone PH, Lalies MD, Hudson AL. Quetiapine and Buspirone Both Elevate Cortical Levels of Noradrenaline and Dopamine In vivo, but Do Not have Synergistic Effects. Front Psychiatry 2012;3:82.

20. Sümegi A. Quetiapin in bipolar disorders. Neuropsychopharmacol Hung 2008;10:281-291.

21. Stahl SM, Lee-Zimmerman C, Cartwright S, Morrissette DA. Serotonergic drugs for depression and beyond. Curr Drug Targets 2013;14:578-585.

22. Hauge-Evans AC, Reers C, Kerby A, Franklin Z, Amisten $\mathrm{S}$, King AJ, et al. Effect of hyperglycaemia on muscarinic M3 receptor expression and secretory sensitivity to cholinergic receptor activation in islets. Diabetes Obes Metab 2014;16:947-956.

23. Locklear JC, Alemayehu B, Brody RS, Chavoshi S, Tunceli $\mathrm{O}$, Kern D, et al. Treatment patterns, healthcare resource utilization and costs in patients with bipolar disorder, newly treated with extended release or immediate release quetiapine fumarate using US healthcare administrative claims data. Clin Ther 2013;35:1923-1932.

24. Bortnick B, El-Khalili N, Banov M, Adson D, Datto C, Raines $\mathrm{S}$, et al. Efficacy and tolerability of extended release quetiapine fumarate (quetiapine XR) monotherapy in major depressive disorder: a placebo-controlled, randomized study. $J$ Affect Disord 2011;128:83-94.

25. Meltzer HY. Update on typical and atypical antipsychotic drugs. Annu Rev Med 2013;64:393-406.

26. Gao K, Wu R, Kemp DE, Chen J, Karberg E, Conroy C, et al. Efficacy and safety of quetiapine-XR as monotherapy or adjunctive therapy to a mood stabilizer in acute bipolar depression with generalized anxiety disorder and other comorbidities: a randomized, placebo-controlled trial. J Clin Psychiatry 2014; 75:1062-1068.

27. Singh J, Chen G, Canuso CM. Antipsychotics in the treatment of bipolar disorder. Handb Exp Pharmacol 2012;(212): 187-212.

28. Sanford M, Keating GM. Quetiapine: a review of its use in the management of bipolar depression. CNS Drugs 2012;26: 
435-460.

29. Yatham LN, Goldstein JM, Vieta E, Bowden CL, Grunze $\mathrm{H}$, Post RM, et al. Atypical antipsychotics in bipolar depression: potential mechanisms of action. J Clin Psychiatry 2005;66 Suppl 5:40-48.

30. Seo MS, Scarr E, Lai CY, Dean B. Potential molecular and cellular mechanism of psychotropic drugs. Clin Psychopharmacol Neurosci 2014;12:94-110.

31. Malhi GS, Tanious M. Optimal frequency of lithium administration in the treatment of bipolar disorder: clinical and dosing considerations. CNS Drugs 2011;25:289-298.

32. Woodward TC, Tafesse E, Quon P, Lazarus A. Cost effectiveness of adjunctive quetiapine fumarate extendedrelease tablets with mood stabilizers in the maintenance treatment of bipolar I disorder. Pharmacoeconomics 2010; 28:751-764.

33. Sokolski KN, Denson TF. Adjunctive quetiapine in bipolar patients partially responsive to lithium or valproate. Prog Neuropsychopharmacol Biol Psychiatry 2003;27:863-866.

34. Pathak S, Findling RL, Earley WR, Acevedo LD, Stankowski J, Delbello MP. Efficacy and safety of quetiapine in children and adolescents with mania associated with bipolar I disorder: a 3-week, double-blind, placebo-controlled trial. J Clin Psychiatry 2013;74:e100-e109.

35. Vieta E, Mullen J, Brecher M, Paulsson B, Jones M. Quetiapine monotherapy for mania associated with bipolar disorder: combined analysis of two international, doubleblind, randomised, placebo-controlled studies. Curr Med Res Opin 2005;21:923-934.

36. Buckley PF, Paulsson B, Brecher M. Treatment of agitation and aggression in bipolar mania: efficacy of quetiapine. $J$ Affect Disord 2007;100 Suppl 1:S33-S43.

37. Cutler AJ, Datto C, Nordenhem A, Minkwitz M, Acevedo L, Darko D. Extended-release quetiapine as monotherapy for the treatment of adults with acute mania: a randomized, double-blind, 3-week trial. Clin Ther 2011;33:1643-1658.

38. Ketter TA. Acute and maintenance treatments for bipolar depression. J Clin Psychiatry 2014;75:e10.

39. Lev-Ran S, Le Foll B, McKenzie K, George TP, Rehm J. Bipolar disorder and co-occurring cannabis use disorders: characteristics, co-morbidities and clinical correlates. Psychiatry Res 2013;209:459-465.
40. Latalova K, Kamaradova D, Prasko J. Suicide in bipolar disorder: a review. Psychiatr Danub 2014;26:108-114.

41. Young AH, McElroy SL, Bauer M, Philips N, Chang W, Olausson B, et al; EMBOLDEN I (Trial 001) Investigators. A double-blind, placebo-controlled study of quetiapine and lithium monotherapy in adults in the acute phase of bipolar depression (EMBOLDEN I). J Clin Psychiatry 2010;71:150162.

42. McElroy SL, Weisler RH, Chang W, Olausson B, Paulsson B, Brecher M, et al; EMBOLDEN II (Trial D1447C00134) Investigators. A double-blind, placebo-controlled study of quetiapine and paroxetine as monotherapy in adults with bipolar depression (EMBOLDEN II). J Clin Psychiatry 2010;71:163-174.

43. Suppes T, Datto C, Minkwitz M, Nordenhem A, Walker C, Darko D. Effectiveness of the extended release formulation of quetiapine as monotherapy for the treatment of acute bipolar depression. J Affect Disord 2010;121:106-115.

44. Suttajit S, Srisurapanont M, Maneeton N, Maneeton B. Quetiapine for acute bipolar depression: a systematic review and meta-analysis. Drug Des Devel Ther 2014;8:827-838.

45. Suppes T, Vieta E, Gustafsson U, Ekholm B. Maintenance treatment with quetiapine when combined with either lithium or divalproex in bipolar I disorder: analysis of two large randomized, placebo-controlled trials. Depress Anxiety 2013;30:1089-1098.

46. Weisler RH, Nolen WA, Neijber A, Hellqvist A, Paulsson B; Trial 144 Study Investigators. Continuation of quetiapine versus switching to placebo or lithium for maintenance treatment of bipolar I disorder (Trial 144: a randomized controlled study). J Clin Psychiatry 2011;72:1452-1464.

47. Young AH, McElroy SL, Olausson B, Paulsson B; Embolden I (D1447C00001); Embolden II (D1447C00134) Investigators. A randomised, placebo-controlled 52-week trial of continued quetiapine treatment in recently depressed patients with bipolar I and bipolar II disorder. World J Biol Psychiatry 2014;15:96-112.

48. Vieta E, Suppes T, Ekholm B, Udd M, Gustafsson U. Long-term efficacy of quetiapine in combination with lithium or divalproex on mixed symptoms in bipolar I disorder. $J$ Affect Disord 2012;142:36-44. 